\title{
Packet Forwarding with Minimum Energy Consumption in Body Area Sensor Networks
}

\author{
Cheng Guo, R. Venkatesha Prasad and Martin Jacobsson \\ Faculty of Electrical Engineering, Mathematics and Computer Science \\ Delft University of Technology, Mekelweg 4, 2600 GA Delft, The Netherlands \\ Email: \{c.guo, r.r.venkateshaprasad, m.jacobsson\}@tudelft.nl
}

\begin{abstract}
Body Area Sensor Networks (BASNs) are powered by batteries and hence energy is a scarce resource. Thus communication protocols in BASN need to be energy efficient. This paper addresses this problem by proposing a Minimum Energy Packet Forwarding Protocol (MEPF). First, we analyze the tradeoff of energy consumption between lower transmission power and packet retransmission. Then we propose the transmission power control part of MEPF. It transmits a packet using the minimum transmission power that can guarantee a high packet reception rate. Unlike the previous work, we not only need to adjust the power of the forward transmission but also the ACKs, which further reduces the consumption. MEPF outstands from the earlier works by also retransmitting lost packets when a link is good enough. The quality of the link is dynamically judged by a machine learning algorithm which also considers the limited buffer space in a sensor node. By MEPF, the energy consumption to forward a packet is minimized without sacrificing the packet reception ratio. Experimental results show quantitatively that MEPF save significant amounts of energy.
\end{abstract}

\section{INTRODUCTION}

Body Area Sensor Networks (BASN) consist of a number of devices equipped with sensors, micro-controllers with radio components embedded in them. They benefit patients and senior citizens by providing them more accurate care. The devices monitor important parameters, such as body temperature, momentum, glucose levels, blood pressure or heart rate of the person who wears them. They can help patients return to their normal life at home with their physical conditions closely monitored by doctors remotely. Devices in such networks normally have limited memory, energy and computational power. Since energy is a scarce resource, how to forward a packet from a sender to a receiver with minimum amount of energy is a challenge in such networks.

Forwarding packets efficiently and reliably is the primary concern. Energy can be saved from transmitting a packet with the lowest possible transmission power and only attempting to retransmit a lost packet when a link is good enough. Thus we try to explore the problem by estimating the Packet Receiving Ratio (PRR) and tune the transmission power as well as retransmit smartly. We denote the former part as Transmission Power Control (TPC) and the latter part is denoted as smart Automatic Repeat reQuest (ARQ). This paper's contribution is two folds. Firstly, it considers minimum energy packet forwarding as a whole, by identifying the trade-off of TPC saving and retransmission energy consumption. Secondly, it proposes the lightweight solutions for not only transmission
TABLE I

MAJOR COMMERCIALLY AVAILABLE WSN PLATFORMS AND THEIR PARAMETERS

\begin{tabular}{c|c|c|c}
\hline Platforms & Processor & RAM & Programming ROM \\
\hline \hline TMote & $8 \mathrm{MHz} 16 \mathrm{bit}$ & $10 \mathrm{kB}$ & $48 \mathrm{kB}$ \\
Mica2 & $16 \mathrm{MHz} 8 \mathrm{bit}$ & $4 \mathrm{kB}$ & $128 \mathrm{kB}$ \\
IRIS & $16 \mathrm{MHz} 8 \mathrm{bit}$ & $8 \mathrm{kB}$ & $128 \mathrm{kB}$ \\
BTNode & $8 \mathrm{MHz} 8 \mathrm{bit}$ & $4 \mathrm{kB}$ & $128 \mathrm{kB}$ \\
\hline
\end{tabular}

power control but also ACK power control as well as a smart ARQ.

We also note here that the energy is not the only limited resource in sensor networks, computational power and memory also need to be taken into account. For example, we list the following four major commercial WSN platforms, i.e. Tmote [1], Mica2 [2], IRIS [3] and BTNode [4] in Table I. From the table, we can see that most of the platforms have micro processors of limited computational ability, thus the protocol designed should have low complexity. Moreover, the RAM and ROM are so small that they quickly fill up. Therefore, the design of an energy efficient protocol should not only focus on the performance, such as delivery ratio and energy consumption, but also the feasibility of such a protocol.

After reviewing briefly the related work in the literature in Section II, we introduce the design of the proposed protocol, Minimum Energy Packet Forward (MEPF), in Section III. In Section IV, we show the performance of the MEPF using experimental data. We conclude the paper in Section V.

\section{RELATED WORK}

APTC [5] outstands from the research in TPC with its first investigation of packet-level adjustment of transmission power. Unlike previous works which either try to tune the transmission power for optimized topology or fix a transmission power for a specific neighbor, APTC focuses on the transmission power control on a per packet level. The authors firstly investigate the temporal-spatial impact on packet delivery ratio by experiments in three different scenarios. With this knowledge, they propose to approximate the transmission power versus RSSI relation with a linear function. Then the parameters in the function are decided by a least square approximation. The realization of APTC in practice relies on a two-phase configuration of the parameters. Nodes in the first phase send beacons in various powers to provide neighbors 
with the RSSIs for configuring the parameters for different transmission powers. In the second phase, which is the runtime of a deployment, a part of the parameters are fine-tuned with the feedbacks of data packet transmissions. However, in designing APTC, the authors only considered the long term temporal impact of the environment, such as temperature and humidity variations or rain. The more challenging scenarios, in which nodes may be mobile or people move around the nodes and cause fading, are not taken into consideration. The fine-tuning algorithm in such cases may not be fast enough to adapt to the environmental changes and thus fail to predict the relation between RSSI and packet delivery ratio.

The authors of [6] shifted the research scenario to BASNs. They showed the fluctuation of wireless links in such a scenario. Compared to [5], the authors set upper and lower thresholds to decide whether to adjust transmission power or not. Two schemes, conservative and aggressive, were proposed. The differences are as the name implies. The conservative scheme is more reluctant to decrease the transmission power and increases to maximum transmission power when it detects the RSSI to be lower than the lower bound. The aggressive scheme only doubles the transmission power when a RSSI lower than the lower bound is found. By experimental results, the authors showed that the conservative and aggressive schemes save $9 \%$ and $25 \%$ compared to the use of maximum transmission power respectively where, according to the authors, the optimum TPC protocol may save up to $35 \%$. A disadvantage of the proposed schemes is that the parameters, which can influence the performance of the schemes a lot, have to be decided arbitrarily since an online learning mechanism is absent. In case the schemes are used in different environments, finding a set of optimal parameters for all the environments may be very difficult, if possible. Like [5], the authors also did not count the energy consumption of retransmissions.

Similar to [5], [7] is a paper with rich implementation details and experimental results. However, the difference is that the authors of [7] proposed to use packet reception rate instead of RSSI as the link quality indicator to control the transmission power. Their protocol is quite straight forward. A desired threshold of PRR is set. Once the actual ratio in a historical sliding packet reception window is lower than the threshold, a node will increase the transmission power if the node is not suffering a high contention or decrease it otherwise. The authors showed that the proposed protocol ART outperformed maximum power transmission in both energy consumption and PRR. However, we must note that the PRR outperforming was in a high contention environment, which means that the medium is most probably saturated with 802.15.4 traffics. In fact, it is not easy to find an application which may saturate the medium except multimedia WSNs. In a low-duty WSN, in case that a packet is sent every minute, the link quality indicator, PRR, in ART may not work well since the extreme low sampling rate of the link, hence the adjustment of transmission power may not be correct and more energy can be consumed.

Authors in [8], [9] have looked into the trade-off between energy consumption and throughput. They modeled the throughput and energy consumption using a Markov model. Then they proposed an easy probing based approach such that whenever an ACK is missed, the transmitting node switches to a probing mode in which only pilot packets are sent periodically until an ACK is received. The idea is to reduce the energy consumption by probing link quality with small pilot packets. However, this protocol assumes that: 1) the channel can be modeled precisely with a Markov chain; 2) the buffer size is unbound; and 3) the transmitter has a lot of packets to be sent. Thus, the pilot packets are sent with a much lower rate than the data packets. The aim is to conserve their energy and transmit the important changes in the environment. However, in a real WSN, say, monitoring environmental parameters, these assumptions may not be true. In fact, most of the WSNs are not designed for the heavy traffic.

\section{The Minimum Energy PACKet Forwarding PROTOCOL}

\section{A. Trade-off between transmission power and packet loss}

To forward a packet with minimum amount of energy, we have to timely estimate the PRR of a link so that the minimum transmission can be achieved. This keeps a the PRR higher. Further, a lost packet can be retransmitted when the PRR is relatively high. Since nodes in a BASN is powered by batteries, we can not probe the link quality by sending pilot packets like in other wireless networks, such as 802.11. The energy that we save from a lower transmission power is not enough to compensate the energy consumed by the pilot packets. Therefore, we use another source, RSSI, to estimate the PRR.

In MEPF, the sender makes decisions of adjusting the transmission power and when to retransmit a packet. It acquires the RSSI by asking the receiver to piggyback it in its acknowledgment. In [10]-[12], it is observed that there is a strong correlation between PRR and RSSI when RSSI is high. We also confirm the same observation with our measurements. Fig. 1 shows the RSSI to PRR mapping in a mobile experiment, in which the receiver was fixed and the sender was moving to and away from the receiver. Both the sender and the receiver used CC2420 transceiver [13]. Each packet was 44 bytes long and the data were collected from an experiment of 1 hours long. The mapping tells us what the PRR is in a slot in which the RSSI is collected. We can see that when the RSSI is higher than $-85 \mathrm{dBm}$, RSSI can be precisely mapped to the PRR, which is close to 1 , with a small variation. Since we want either to adjust the transmission power to have a high PRR or find a link state with a higher PRR, an indication of a good link state is enough in both the cases. Using the high RSSI values to estimate the PRR serves these purposes well.

Unlike previous work, which either focus on TPC or ARQ, we consider the problem of minimum energy packet forwarding as a whole. Lower transmission power may increase the packet loss on a link. Since we have to retransmit the lost packets, the energy saved by decreasing the transmission power may not be enough to compensate those consumed by 


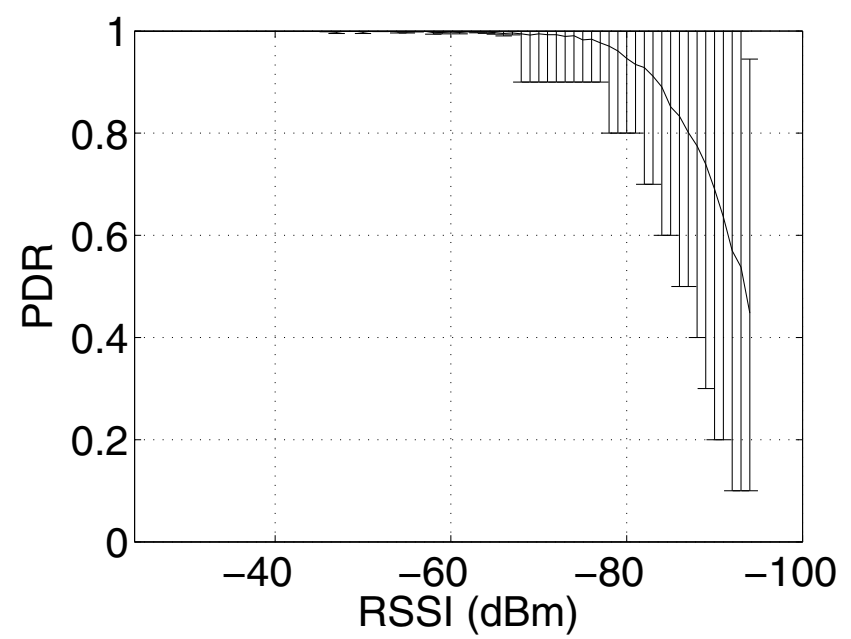

Fig. 1. Correlation between RSSI and PRR. The solid line is the average and the bars indicate the $90 \%$ percentile

retransmitting the packets. Thus, we have a trade-off between transmission power and packet loss.

Let us take the widely used hardware CC2420 [13] as an example to analyze the energy saving and consumption of lower transmission power and retransmission respectively. Table II shows the power level, output power and current consumption of transmitting in CC2420. We can see that when we decrease the power level from 31 , each step of 4 , the output power decrease from $0 \mathrm{dBm}$ by $1,2,2,2,3,5,10$ $\mathrm{dBm}$ per step respectively. The current consumption decreases approximately 1.3 to $1.4 \mathrm{~mA}$ per step except the first step of $0.9 \mathrm{~mA}$. The insight from the above observation is that we have to take extra care when decreasing the power level to the last two levels, which does not save more energy but degrade the output power a lot. The current consumption of receiving is $19.7 \mathrm{~mA}$. The voltage of transmitting and receiving is same as 1.8 volt as in $\mathrm{CC} 2420$.

We assume that (1) a lost packet is retransmitted and acknowledged with the highest power level 31; (2) transmitting an ACK takes half amount of time of transmitting a data packet which is normalized as 44 bytes; (3) a node is awake only when it is transmitting or receiving thus no energy is consumed by idle listening; (4) data rate is $240 \mathrm{~kb} / \mathrm{s}$ and (5) energy consumed in other components of a node is negligible. We can deduce the normalized power consumption of retransmitting a packet is $E_{\text {retr }}=(17.4 * 1+19.7 * 1+17.4 * 0.5+19.7 *$ $0.5) * 1.8 * \frac{44 * 8}{2.4 * 10^{5}}=0.147 \mathrm{~mJ}$, the maximal power saving from reducing one level of power is $E_{\text {save }}=1.8 * 1.4 *$ $\frac{44 * 8}{2.4 * 10^{5}}=0.0037 \mathrm{~mJ}$. Therefore 1 retransmission would use up the energy saved from 40 packets transmitted with one level lower power. The insight from this observation is that we would rather use the transmission power one level higher than risking to loose one packet.
TABLE II

OUTPUT POWER FOR THE CC2420 [13]

\begin{tabular}{c|c|c}
\hline Power Level & Output Power $(\mathrm{dBm})$ & Current Consumption $(\mathrm{mA})$ \\
\hline 31 & 0 & 17.4 \\
\hline 27 & -1 & 16.5 \\
\hline 23 & -3 & 15.2 \\
\hline 19 & -5 & 13.9 \\
\hline 15 & -7 & 12.5 \\
\hline 11 & -10 & 11.2 \\
\hline 7 & -15 & 9.9 \\
\hline 3 & -25 & 8.5 \\
\hline
\end{tabular}

\section{B. Transmission Power Control}

To understand the relation between transmitting and receiving powers, we conducted an experiment in a corridor of the TU Delft Wireless and Mobile Communications Lab, which has offices on both sides. The transceiver pair was kept in the middle of the corridor, thus there was LOS between them. We used t-mote sky sensor motes with 2.4 GHz IEEE 802.15.4 compliant Texas Instruments CC2420 transceivers [13]. The transceiver provides a RSSI in the range of $100 \mathrm{dBm}$ for every received packet, which is the received signal strength averaged over the first eight symbol periods. The IEEE 802.15.4 packet header is 17 bytes and the payload is set to 27 bytes, thus a packet is 44 bytes long. An ACK has a 4 bytes sequence number and an 1 byte RSSI value as payload, thus an acknowledgment packet is 22 bytes. We consider this length as typical for WSN packets. The distances between the two transceivers were 2, 4, 816,32 and 50 meters. In each round, the transmitter firstly sent 20 packets per second with the maximum power level and then shifted to the next lower one and so on until the lowest one. In the same distance, the experiment runs for 100 rounds in total. For each packet transmission, the receiver acknowledges with the same power level as the transmitter. Fig. 2 shows the output power and RSSI difference when we grade down the transmission power. We can see that, in general, the degrading of RSSI is more than that of output power when we reduce the transmission power. All the curves in the figure follow the same trend.

We use a packet level TPC in MEPF because of the temporal characteristic of links, which will be illustrated in the next section. A link may be in a good state at one moment in time but may degrade to a very low PRR a minute later due to shadowing or interference. To use the minimum transmission power, we need a model to predict the RSSI while shifting transmission power. However, unlike in [5], we do not maintain an equation for every power level, which increases the complexity of a protocol. Instead, as we can see in Fig. 2, all the curves in the figure follow the same trend and overlap with each other more or less. Thus we use only one equation for all power levels. As we observed at the beginning of the section, we would rather use the transmission power one level higher than risking to loose a packet. Therefore, while decreasing the transmission power, the model should predict the RSSI decrement more prominently compared to the other curves in the figure and while increasing, the predictive increment 


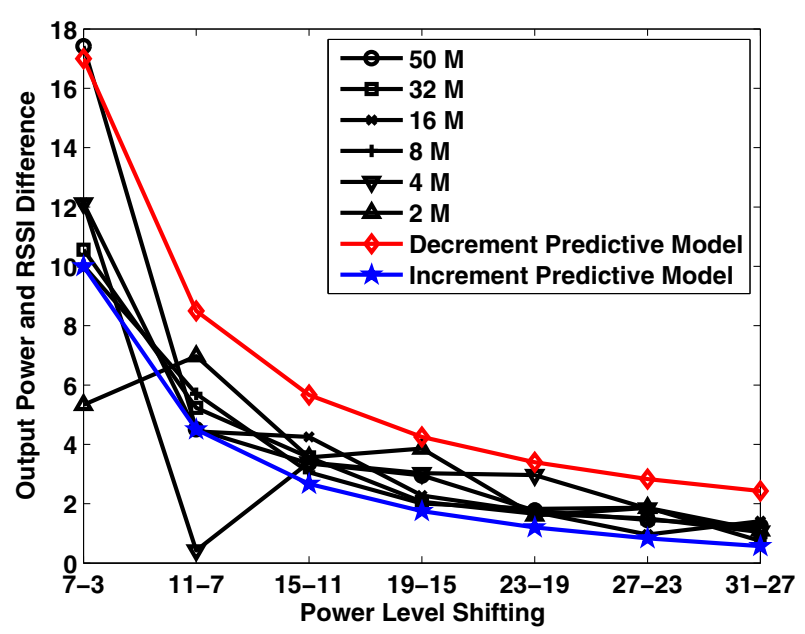

Fig. 2. RSSI difference when lowering the transmission power (“-” means shifting from one power level to another)

should be less than all the curves. MEPF uses the equation $R S S I=\frac{68}{\text { power level }+1}$ to predict the RSSI while decreasing the transmission power and $R S S I=\frac{44}{\text { power level+1 }}+1$ otherwise. The two equations are derived from curve fitting in Fig. 2. The curve of the decrement predictive model is above all the other curves and the increment model is below the other curves.

A receiver normally has a sensitivity. A packet can be received if the receiving power is above the sensitivity level. Due to the fluctuation of a link, the receiving power has to be a few $\mathrm{dB}$ higher than the sensitivity to guarantee a high PRR. So a threshold should be set. Adjustment of transmission power should make sure the RSSI is above the threshold. For example, the sensitivity of the CC2420 is $-94 \mathrm{dBm}$ [13] and we set the threshold in MEPF as $-85 \mathrm{dBm}$. Again, we are more cautious while decreasing power than increasing it. When RSSI is above $-85 \mathrm{dBm}$, MEPF only decreases the transmission power by one level but when it is below the threshold, we use the increment predictive model to decide which power level can increase the RSSI above the threshold. In the case that a packet is lost, no RSSI can be collected and we increase the transmission power to the maximum level immediately.

The TPC in MEPF has another feature, it also adjusts the transmission power of ACKs. With the same consideration of reducing packet loss, we set the transmission power of ACKs one level higher than that of the data packet. The purpose of using one level higher power is to guarantee a higher PRR in the asymmetric links when the reverse path is weaker than the forward one.

\section{Automatic Repeat Request}

When a packet is lost, the link is normally in a bad state, retransmitting immediately, as in the Stop\&Wait ARQ, may not deliver the packet but could waste more energy. Thus, MEPF stores the lost packet in a buffer and wait until the link is good enough to retransmit. The link quality is also estimated by RSSI. If the link stays in the bad state for a long time, the buffer may be full, MEPF would have to do its best by retransmitting the buffer head for a few times (3 in the current MEPF implementation) before dropping it. To avoid such blind retransmissions, we have to prevent the buffer from becoming full. Therefore, we can not set a hard threshold to judge the quality of a link like the one used to adjust transmission powers. In the case that we have a hard threshold as a criterion, say for example when RSSI is higher than $-80 \mathrm{dBm}$, the sender may not have a chance to send the buffered packets in case the transceiver pair was deployed far apart or a lot of multi-path or interference is present and thus causing the RSSI to never be as high as $-80 \mathrm{dBm}$. Another important aspect is that mere RSSI being low will not preclude a proper transmission at all the times. Sometimes packets do get across even if RSSI is low. So instead of a hard threshold, we use a soft threshold by looking into the past received RSSIs. If the just received RSSI is among the $T$ percent of highest RSSIs in the past, then we consider the channel as relatively usable. It may be the case that even with a RSSI among the $T$ percent of the highest, the delivery ratio may not be enough. However, the past measurements tell us that RSSI is not always as high as one can expect it to be. So the node should take the opportunity to transmit all the buffered packets.

The soft threshold is adjusted by a simple machine learning mechanism. If the buffer is full and we have to blindly retransmit the buffer head, we may have set the threshold too high with respect to the link, thus MEPF fails to "find" a good state. So we reduce the threshold by $m$ (with $m=3$ in the current implementation). On the contrary, if a retransmission fails when the RSSI is above the current threshold, it means that the threshold is set too low, thus MEPF is misled to retransmit buffered packets when the link is not good. In this case, we should increase the threshold by $n$, so that MEPF uses a tighter criterion to "find" a good link. Since we blindly retransmit a packet 3 times before dropping it. $n$ is 3 times smaller than $m$ (with $n=1$ in current implementation). Stop\&Wait mechanism is used in retransmitting. After each retransmission, MEPF waits for an acknowledgment and judges the link quality before the next retransmission.

The TPC and ARQ parts in MEPF work independently to each other. They individually make their decisions. However, while the TPC tries to use the power which can keep the RSSI above $-85 \mathrm{~dB}$, which does not decrease the PRR, it makes ARQ an easier task. The ARQ may use a lower power to retransmit a packet when a link is in a good state. The performance of MEPF will be shown in the next section.

\section{PERFormance OF MEPF}

To evaluate the performance of MEPF, we carried out experiments. Instead of implementing the protocol in an experimental testbed, we ran some measurements and recorded the PRRs and RSSIs in different scenarios and then used the recorded data to run a simulation. This measurement data 
driven simulation enables us to repeat the experiment in the same environment to collect statistical results.

The hardware used in the measurement was the same as introduced in Section III. However, we changed the experimental place from the corridor to our canteen where people move around between nodes. Therefore, we expected a heavy variation of link quality along with time. The sender and receiver were first kept 10 meters apart, then 20 meters apart, and finally 30 meters apart. The application scenario of this deployment is that a person with wearable sensors moves at his home or a meeting room and stays at each place for some time. In total 678 packets were sent. We expect a very high PRR when the nodes are 10 meters apart, thus TPC can show its ability of using minimum transmission power. When the nodes are 30 meters apart, TPC is more cautious to use a lower transmission power and ARQ smartly decide when to retransmit a packet.

In all the experiments, we operated in a $2.4 \mathrm{GHz}$ range absent from WLAN activity to minimize the interference from other wireless systems. The buffer size in the ARQ mechanism is 40 , which occupies 720 bytes in RAM. We saved the last 100 RSSIs as the history to which we apply the soft threshold.

\section{A. Simulation Results}

We want to evaluate the performance of MEPF with a few metrics, namely power level, Retransmission Ratio (RR), dropped packets and energy consumption. Power level is the transmission power index used to transmit packets. RR is defined as

$$
R R=\frac{\text { Retransmission number }}{\text { Lost packet number }} .
$$

If a sender transmits 10 packets, two of them are not received (lost packet) and the sender retransmits four times (retransmission number) to deliver these packets then the $\mathrm{RR}$ is 2 . We note that the optimum ARQ protocol should retransmit a lost packet only once and thus has a RR equal to 1 . Dropped packets are those that can not be delivered even after a maximum number of retransmissions. Energy consumption is the total energy spent to deliver all the packets. MEPF is compared with three protocols, namely plain, TPC only and $A R Q$ only. The first one does not have TPC nor smart ARQ and the second and the third one does not have TPC and smart ARQ respectively.

Firstly, we compare the optimum transmission power derived from the simulation data and the one selected by MEPF. We define the optimum transmission power as the power which is just enough to deliver packets with a PRR higher than $95 \%$. It is the theoretical upper bound which can be reached by TPC mechanisms. Fig. 3 shows the comparison. We can see that in general, the optimum transmission index is lower than the one selected by MEPF. On an average, the optimum transmission power is 11.39 and the MEPF uses 17.14, which is more than a level higher than the optimum one. In the three locations, the first one shows the largest difference between the optimum and MEPF. Because in the location, the sender and receiver

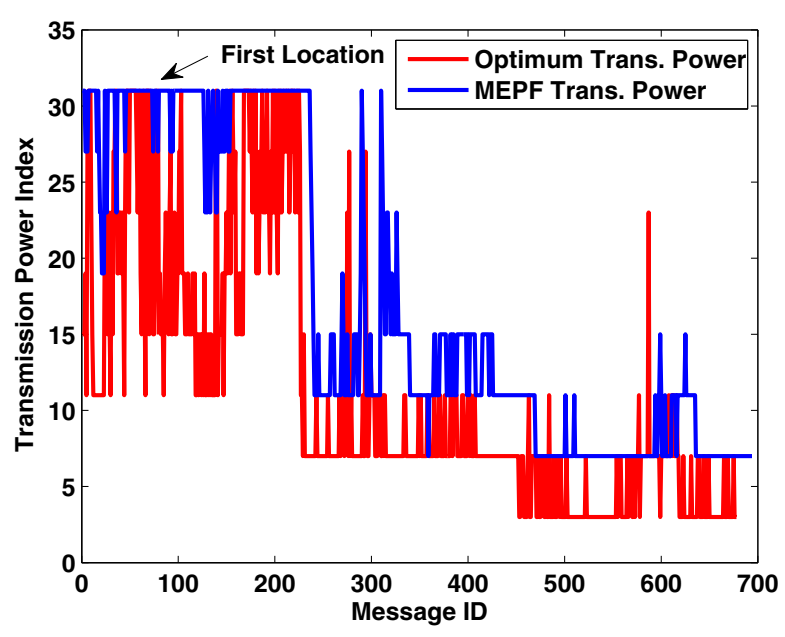

Fig. 3. Transmission power comparison

is placed far apart, the RSSI is usually lower than the hard threshold so that MEPF always uses the highest transmission power. In the other two locations, MEPF generally used a level higher transmission power than the optimum one. By further investigation, we found that the RSSI is around $-90 \mathrm{dBm}$ with the optimum transmission power and MEPF leads to a RSSI around $-85 \mathrm{dBm}$. However, lowering the hard threshold increases packet loss.

Fig. 4 shows the RR and the number of dropped packets. Since only the ARQ mechanism effects the performance of these two metrics, we compared MEPF only with the plain protocol. The bars in the figure show standard variations. The RR in MEPF and the plain protocol are 1 and 2.39 respectively. The MEPF has the optimum RR, which means it retransmits the lost packet only when the link is good enough and thus each lost packet is delivered by one retransmission. Contrarily, a normal ARQ protocol retransmits each lost packet 2.39 times on an average, which consumes more energy. Since MEPF retransmits smartly, it does not drop any packet in the whole of the simulation. Although the plain protocol spends more energy on retransmissions, it drops 4.67 packets on average. The packets are dropped only in the first location, which is farthest from the receiver. We can imagine that if we keep the sender at the location for longer time, the dropped packet would have increased along with time. Consequently, more energy would have been wasted.

Finally, we check the energy consumption of the four protocols in Fig. 5. At least, an optimum protocol consumes $84.42 \mathrm{~mJ}$ energy. The optimum protocol transmits with optimum energy levels presented above and no packet is lost and hence no packet has to be retransmitted. Contrarily, the plain protocol, which transmits with maximum transmission level and retransmits with a normal Stop\&Wait protocol, consumes $103.71 \dot{\mathrm{m} J}$ energy. The difference with the optimum protocol is the room that we can improve. A protocol which has MEPF's TPC mechanism but no smart ARQ protocol 


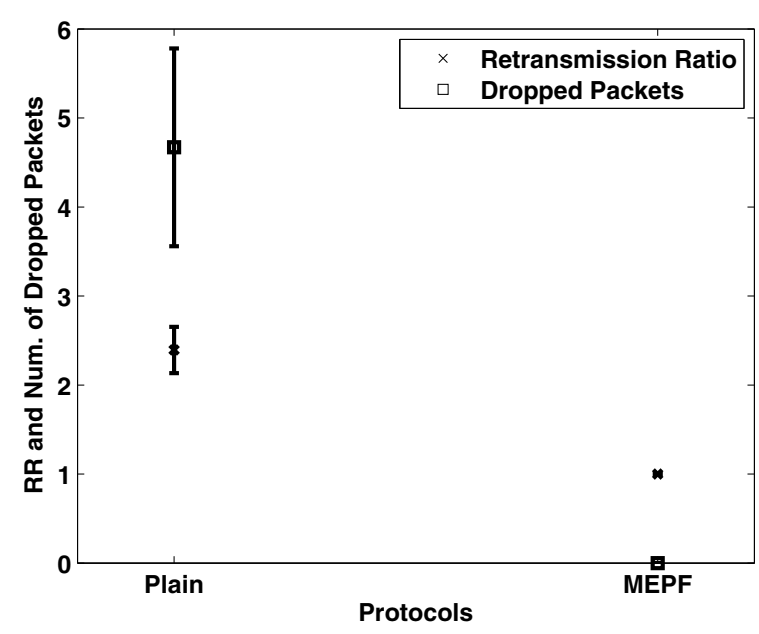

Fig. 4. Retransmission ratio and number of dropped packets

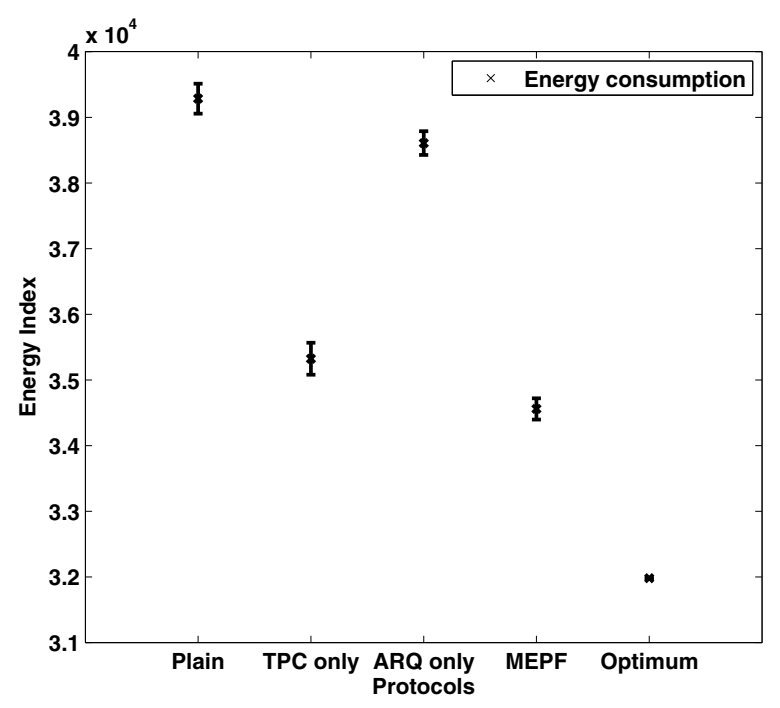

Fig. 5. Energy consumption

consumes $93.25 \mathrm{~mJ}$ energy, which is $10.46 \dot{\mathrm{m} J}$ less than the plain protocol. Thus in this scenario, we can save about $10 \%$ of the energy consumption by the proposed TPC mechanism. Alternatively, the $A R Q$ only protocol consumes $101.92 \dot{\mathrm{m} J}$ energy which is $1.79 \dot{\mathrm{m} J}$ less than the plain protocol. Thus $1.7 \%$ of energy is saved. The small amount of saving is due to the fact that the experimental scenario was not very challenging with respect to the packet loss, only a bit more than 10 packets was lost in the farthest location. Therefore, TPC can save more energy than a smart ARQ. We can imagine that in a weak link, the smart ARQ mechanism would have saved more. The full

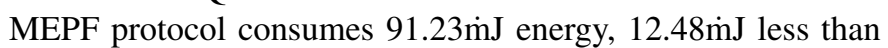
the plain protocol, which corresponds to $12 \%$ saved energy. Although the amount is not large, we note that the energy is saved without any extra communication overhead, only a bit extra memory and computation is spent.

\section{Conclusions}

In this paper, we introduced Minimum Energy Packet Forwarding Protocol (MEPF), which is a TPC protocol designed for Body Area Sensor Networks (BASNs). We analyzed the trade-off of energy consumption between lower transmission power and packet retransmission and used this in the design of MEPF. MEPF transmits a packet using the minimum transmission power that can guarantee a high packet reception rate. At the same time, retransmissions are postponed until the link is good enough. The quality of the link is dynamically judged by a machine learning algorithm, designed based on the limited capabilities of typical BASN devices.

Using simulations based on real measurements from two 802.15.4 devices, we demonstrated the energy saving of MEPF. We showed that MEPF's TPC mechanism saved 10\% in our scenario and that the proposed ARQ saved $1.7 \%$. When combined, they saved $12 \%$ of the energy consumption. In the future, we plan to do more experiments to investigate the energy saving performance of MEPF in many more and different scenarios.

\section{REFERENCES}

[1] (2006) Tmote sky datasheet. Moteiv Corporation. [Online]. Available: http://www.moteiv.com/products/docs/tmote-sky-datasheet.pdf

[2] (2008) MICA2 datasheet. Crossbow. [Online]. Available: http://www.xbow.com/Products/Product_pdf_files/Wireless_pdf/ MICA2_Datasheet.pdf

[3] (2008) Iris datasheet. Crossbow. [Online]. Available: http://www.xbow. com/Products/Product_pdf_files/Wireless_pdf/IRIS_Datasheet.pdf

[4] (2007) Btnode rev3.24 product brief. ETHZ. [Online]. Available: http:// www.btnode.ethz.ch/pub/uploads/Main/btnode_rev3.24_productbrief.pdf

[5] S. Lin, J. Zhang, G. Zhou, L. Gu, J. A. Stankovic, and T. He, "Atpc: adaptive transmission power control for wireless sensor networks," in SenSys '06: Proceedings of the 4th international conference on Embedded networked sensor systems. New York, NY, USA: ACM, 2006, pp. 223-236.

[6] S. Xiao, V. Sivaraman, and A. Burdett, "Adapting radio transmit power in wireless body area sensor networks," in BodyNets '08: Proceedings of the ICST 3rd international conference on Body area networks. ICST, Brussels, Belgium, Belgium: ICST (Institute for Computer Sciences, Social-Informatics and Telecommunications Engineering), 2008, pp. 18.

[7] G. Hackmann, O. Chipara, and C. Lu, "Robust topology control for indoor wireless sensor networks," in SenSys '08: Proceedings of the 6th ACM conference on Embedded network sensor systems. New York, NY, USA: ACM, 2008, pp. 57-70.

[8] M. Zorzi and R. R. Rao, "Error control and energy consumption in communications for nomadic computing," IEEE Trans. Comput., vol. 46, pp. 279-289, 1997.

[9] — "Energy constrained error control for wireless channels," IEEE Personal Commun. Mag., vol. 4, pp. 27-33, 1997.

[10] D. Aguayo, J. Bicket, S. Biswas, G. Judd, and R. Morris, "Linklevel measurements from an $802.11 \mathrm{~b}$ mesh network," in Proc. ACM SIGCOMM'04, Portland, OR, USA, Aug. 30 - Sep. 3, 2004.

[11] K. Srinivasan, P. Dutta, A. Tavakoli, and P. Levis, "Some implications of low power wireless to IP networking," in Proc. HotNets-V, Irvine, CA, USA, Nov. 29-30, 2006.

[12] J. Zhao and R. Govindan, "Understanding packet delivery performance in dense wireless sensor networks," in Proc. ACM SenSys'03, Los Angeles, CA, USA, Nov. 5-7, 2003.

[13] (2008) CC2420 RF transceiver datasheet. Texas Instrumets. [Online]. Available: http://focus.ti.com/docs/prod/folders/print/cc2420.html 